\title{
LA CONTABILIDAD Y LA GESTIÓN DEL CONOCIMIENTO
}

\author{
C.P.C. Miguel Diaz Inchicaqui*
}

\begin{abstract}
RESUMEN
El presente artículo titulado: "La Información Contable y la Gestión del Conocimiento" analiza la problemática actual de la ciencia contable como parte del sistema de información empresarial que manejan los gestores de negocios y su relación con la gestión del conocimiento, la misma que consideramos es fundamental para la empresa moderna que busca continuamente como objetivo corporativo ser competitiva en el mercado o industria en el que desarrolla sus actividades empresariales. Asimismo, es importante señalar que todos los profesionales contables que brindamos asesoria de negocios debemos conocer esta relación existente entre la contabilidad y la gestión del conocimiento, para reconocer las fortalezas y debilidades de la información que manejamos ya que de ésta dependerá el manejo adecuado y racional de las variables internas y externas, la cual será determinante en la toma de decisiones que realizan los niveles estratégicos de las empresas. En nuestro país la gran mayoría de nuestras empresas aún no han ingresado en el marco de una economía globalizada, por mantener estas particularidades de empresas familiares, sin embargo, si deseamos salir de la recesión económica en la cual estamos inmersos, tendremos que insertar a nuestras empresas en el mundo globalizado donde la competitividad y la búsqueda del beneficio económico son las características de la empresa moderna en la economía del conocimiento.
\end{abstract}

\section{INTRODUCCIÓN}

La profesión contable viene enfrentando un reto importante vinculado con la revisión de las prácticas de exposición de la información financiera útil a los inversionistas para la toma de decisiones, ya que hace cierto tiempo que las organizaciones empresariales se dieron cuenta que sus activos físicos y financieros no tienen la capacidad de generar ventajas competitivas sostenibles en el tiempo, descubriéndose entonces que los activos intangibles son los que aportan verdadero valor a las organizaciones. Pero ¿a qué nos referimos cuando hablamos de activos intangibles?, para dar respuesta a esta interrogante muy genérica, pero importante; definiremos a los activos intangibles como una serie de recursos que pertenecen a la

* Docente Asociado de la Facultad de Ciencias Contables. 
organización, pero que no están valorados desde un punto de vista contable. También son activos intangibles las capacidades que se generan en la organización, cuando los recursos empiezan a trabajar en grupo y coordinadamente muchos profesionales en lugar de capacidades hablan de procesos, o rutinas organizativas. En definitiva un activo intangible es todo aquello que una organización utiliza para crear valor, pero que no está contabilizado en la información que generamos los profesionales contables. Dentro de este grupo de intangibles se sitúan los recursos humanos como el principal activo que sustenta el poder de competitividad de la empresa modema, por la capacidad que tienen éstos de compartir la información, sus experiencias y conocimientos tanto individuales como colectivos.

Como consecuencia del párrafo anterior, en esta oportunidad sostengo que la ciencia contable tendrá que abandonar en un futuro no muy lejano la conversión del costo histórico ya que la información financiera tradicional no identifica las fuentes de valor puestas a trabajar en la actual economia del conocimiento.

\section{LA INFORMACIÓN CONTABLE EN LA ERA DEL CONOCIMIENTO}

Las nuevas necesidades informativas en la Era del Conocimiento, las tecnologías actuales $y$ los cambios que experimenta la Contabilidad deben dar respuesta a las nuevas demandas de información que requieren los usuarios, tales como: Identificar y valorar el capital intelectual de las empresas, para desarrollar la adecuada gestión del conocimiento. Como los gestores de negocios se ubican en el nivel estratégico de las empresas, éstos requieren de cierta información necesaria para realizar una adecuada gestión del conocimiento, tales como:

- Información sobre los riesgos a que está sometida la empresa.

- Los recursos humanos.

- Los activos intelectuales.

- El impacto medioambiental.

- Las impresiones de los clientes sobre la calidad de nuestros productos, etc.

Uno de los recientes debates sobre a dónde debe ir la Contabilidad, trata sobre si debe incluirse el llamado capital intelectual en los estados financieros de las empresas. Esta postura enlaza con las recientes teorias de economía de empresa ligadas a la gestión del conocimiento en la empresa y al éxito que están teniendo las empresas que destacan más por sus activos intangibles como; la imaginación, capacidad de inventiva, grado de satisfacción de los clientes o la motivación de los empleados, etc., que por su activo fijo o su estructura financiera. Es decir, se trata de valorar los recursos intelectuales de las empresas como un activo intangible más, y crear nuevos documentos contables. Es también un área de interés en otros campos como la Sociología por cuanto involucra la gestión de recursos humanos.

Otros usuarios de la información contable como los analistas 
externos manifiestan su decepción ante la información contable suministrada por las empresas y su interés en conocer más sobre los activos intangibles de las mismas. Además, de requerir que la información contable sea oportuna en el tiempo. Hoy en día, información oportuna es información en tiempo real.

Pero ¿el problema de mostrar el valor de las empresas que permita la toma de decisiones de inversión se reduce exclusivamente a corregir las reglas de valuación de sus activos y pasivos? ¿Qué otros temas no son convenientemente recogidos y expuestos en la información financiera y que representan fuente de valor de las empresas? Especialistas en la materia consideran que el modelo de presentación de información financiera que conocemos, es decir los estados financieros tradicionales, fue diseñado para cumplir con los requerimientos de la era industrial. Este modelo, que fue útil en su oportunidad, desafortunadamente, no identifica las fuentes de valor puestas a trabajar en la actual economía del conocimiento y de la comunicación por internet.

En una publicación titulada The Value Reporting Revolution se transmite un mensaje importante: "la práctica tradicional relativa a la información suministrada por las corporaciones es inadecuada porque no recoge la información del mercado y los recursos no financieros que generan valor en las empresas. En la actualidad se aprecia que los inversionistas depositan demasiada confianza en los resultados financieros de corto plazo, práctica que ha contribuido a la volatilidad en los mercados de capitales." ¿Cuál es entonces la solución? A decir de los autores de esta publicación, "la exposición de mayor y mejor información". En la citada publicación se propone un modelo de exposición de información sobre valor, que surge de las necesidades de los inversionistas, y que comprende cuatro elementos interrelacionados, que tomados en conjunto brindan una visión integral de los planes y el desempeño de la empresa. Estos elementos son:

a) Examen del mercado, la conducción de la gerencia sobre la base de la posición competitiva de la empresa y su ambiente externo.

b) Estrategia de valor, explicación de la estrategia de la empresa, incluyendo como intenta crear valor.

c) Administración de valor, resumen de los objetivos de desempeño de la empresa y la evaluación de su consecución.

d) Plataforma de valor, elementos que soportan valor y el desempeño financiero futuro, que incluye al personal, innovación, cadena de suministro, clientes, marcas y reputación.

En este siglo, con la globalización de los negocios y con la velocidad de los cambios tecnológicos, revisiones como la planteada, que pueden parecernos de otra realidad, es preciso sostener que estará gobernándonos en poco tiempo.

En las ciencias contables hoy día la cuestión perentoria es medir y evaluar financieramente activos intangibles tales como: capital intelectual humano, las marcas y las patentes. La relevancia de los activos intangibles ha sido tan 
significativa que ha traido como consecuencia el diseño y formulación de la Norma Internacional de Contabilidad NIC 38-(IAS 38) para la administración de esta herramienta y de manera muy particular su aceptación, valorización y revelación en los estados financieros. Otra de las innovaciones contables del conocimiento es el "Study 7" (IFACFMAC), el cual define algunas diferencias en relación con dicho asunto, debido a que determina la dirección de los nuevos procesos de contabilidad moderna, los cuales están siendo considerados más convenientes como instrumentos de información para la gerencia del capital intelectual humano.

Para incorporar el valor del capital intelectual a los estados financieros que se presenten en primer lugar debemos cumplir con los requisitos establecidos en la NIC 38 , que son los siguientes:

- Ser reconocido como activo intangible de acuerdo con lo descrito en el párrafo 8.

- Identificar el intangible de acuerdo con el párrafo 10 y 11 .

- Debe estar bajo el control de la entidad de acuerdo con los párrafos 13,14, 15, y 16.

- Los intangibles deben otorgar beneficios económicos futuros de acuerdo con el párrafo 17.

Con posterioridad a lo indicado en el párrafo anterior se debe seguir los pasos establecidos en uno de los métodos que se viene administrando en la contabilidad moderna tales como:

- El "Valor Económico Agregado" (EVA) (Economic Value Added) y el "Valor de Mercado Agregado" (MVA) (Market Value Added).
EVA es la utilidad operacional neta después de impuestos (NOPAT) menos un gasto de capital. De acuerdo con este indice si la rentabilidad/retorno sobre el capital de una empresa sobrepasa sus costos de capital, se está creando verdadero valor para los accionistas. Estas medidas forman parte de un abanico más grande denominado "Análisis de Valor de Accionista" (SVA) (Shareholder Value Analysis), que ha representado la corriente más vigorizante de esta materia en la última década. Este indice mezcla tres elementos importantes:

1. Flujo de efectivo,

2. Efectivo medido durante un período dado de tiempo (duración del crecimiento del valor), $\mathrm{y}$

3. Riesgo, de otro modo conocido como costo de capital.

- Navigator de Skandia, que tiene como línea de argumentación de este modelo, es la diferencia entre los valores de las acciones en libros y el valor de mercado, la cual se debe a un conjunto de intangibles que quedan reflejados en la contabilidad tradicional, pero que el mercado lo reconoce como flujo de caja. Este modelo parte de que el valor de mercado de la empresa esta integrado por el capital financiero y el capital intelectual, el cual se descompone en bloques.

- Intellectual Asset Monitor (Sveiby), la medición presenta doble orientación hacia el exterior para informar a los clientes, accionistas y proveedores, y hacia el interior dirigida al equipo directivo para conocer la marcha de la empresa. 
Esta nueva orientación de la contabilidad moderna y su obligación de contabilizar los activos intangibles ha despertado interés hasta del Santo Padre Juan Pablo II, quien en su encíclica "Fides et Ratio", fija la posición de la Iglesia sobre el particular. Otro de los aportes significativos en la contabilidad de los activos intangibles está constituido por lo que se denomina "Modelo de Valoración de Negocios" (Business Assesment Model), el cual se puede decir que tiene implícito una nueva visión del negocio: es necesario ir más allá de los números tradicionales $y$ ayudar a implementar la estrategia, que lo logra por medio de la estructuración de tres jerarquias:

1. Cualidad del liderazgo = administración;

2. Creación de valor $=$ operaciones + desempeño; y

3. Generación de ingresos $=$ negocio central + mercado + competencia.

Los expertos en la contabilidad del conocimiento, entre ellos A. S. Mantilla; definen el valor corporativo como la suma del valor presente de todos los flujos de efectivo y el valor de mercado de los activos nooperacionales. Mientras que el valor de accionista lo define como la suma del valor corporativo más el valor de mercado de todas las deudas. En el aspecto de contabilidad general se ha avanzado mucho más, por cuanto se viene hablando recientemente del "Círculo aprendizajeconocimiento-valor".

\section{EL CONOCIMIENTO EMPRESARIAL}

Cuando planteamos nuestro artículo en primer lugar nos preguntamos: ¿Qué es el conocimiento?, pregunta que ha sido analizada por las mentes más privilegiadas del pensamiento occidental, desde Aristóteles y Platón hasta la actualidad. Recordemos una cita de Platón: "Supongamos ahora que en la mente de cada hombre hay una pajarera con toda suerte de pájaros. Algunos en bandadas aparte de los demás, otros en pequeños grupos, otros a solas, volando de aquí para allá por todas partes... Podemos suponer que los pájaros son tipo de conocimiento y que cuando éramos niños este receptáculo estaba vacío; cada vez que un hombre obtiene y encierra en la jaula una clase de conocimiento, se puede decir que ha aprendido o descubierto la cosa que es el tema del conocimiento; y en esto consiste el saber".

El diccionario define conocimiento como: "El producto o resultado de ser instruido, el conjunto de cosas sobre las que se sabe o que están contenidas en la ciencia". El diccionario acepta que la existencia de conocimiento es muy difícil de observar y reduce su presencia a la detección de sus efectos posteriores. Los conocimientos se almacenan en la persona (o en otro tipo de agentes). Esto hace que sea casi imposible observarlos.

Como vemos existen múltiples definiciones de conocimiento, desde las clásicas y fundamentales como una creencia cierta y justificada, a otras más recientes y pragmáticas como una mezcla de experiencia, valores, información y "saber hacer" que sirve como marco para la incorporación de nuevas experiencias e información, y es útil para la acción. De todo ello, podriamos resumir las características del conocimiento: 
- El conocimiento es personal, en el sentido de que se origina y reside en las personas, que lo asimilan como resultado de su propia experiencia (es decir, de su propio "hacer", ya sea físico o intelectual) y lo incorporan a su acervo personal estando "convencidas" de su significado e implicaciones, articulándolo como un todo organizado que da estructura y significado a sus distintas "piezas".

- Su utilización, que puede repetirse sin que el conocimiento "se consuma" como ocurre con otros bienes fisicos, permite "entender" los fenómenos que las personas perciben (cada una "a su manera", de acuerdo precisamente con lo que su conocimiento implica en un momento determinado), y también "evaluarlos", en el sentido de juzgar la bondad o conveniencia de los mismos para cada una en cada momento.

- Sirve de guía para la acción de las personas, en el sentido de decidir qué hacer en cada momento porque esa acción tiene en general por objetivo mejorar las consecuencias, para cada individuo, de los fenómenos percibidos (incluso cambiândolos si es posible).

Estas caracteristicas convierten al conocimiento, cuando en él se basa la oferta de una empresa en el mercado, en un cimiento sólido para el desarrollo de sus ventajas competitivas. En efecto, en la medida en que es el resultado de la acumulación de experiencias de personas, su imitación es complicada a menos que existan representaciones precisas que permitan su transmisión a otras personas efectiva y eficientemente.
Por lo tanto, conjugando nuestros conceptos más importantes de nuestra investigación definimos "Gestión del Conocimiento", como un "Conjunto de procesos y sistemas que permiten que el Capital Intelectual de una organización aumente de forma significativa, mediante la gestión de sus capacidades de resolución de problemas de forma eficiente (en el menor espacio de tiempo posible), con el objetivo final de generar ventajas competitivas sostenibles en el tiempo". Parece claro que el desarrollo de conocimiento se hace con el objetivo de emplearlo en la consecución de ventajas competitivas sostenibles, no simplemente acumulando conocimiento sin aplicarlo.

\section{LA GESTIÓN DEL CONOCIMIENTO}

Gestionar el Conocimiento viene a ser la gestión de todos los activos intangibles que aportan valor a la organización a la hora de conseguir capacidades 0 competencias esenciales, distintivas. Es por lo tanto, un concepto dinámico, que describe y explica ¿Cuál es la actitud de los empresarios peruanos hacia la Gestión del Conocimiento? Para lo cual, es preciso entender la gestión del conocimiento como una nueva cultura empresarial, situando a los recursos humanos como el vital y más importante en el presente siglo.

La razón principal del tema Gestión del Conocimiento es la mejora de los resultados de la acción de las organizaciones:

- Aumentar la productividad de las empresas y corporaciones.

Aumentar la competitividad. 
Aumentar la capacidad de liderazgo de las organizaciones.

Incrementar las oportunidades de negocio.

El tema "Gestión del Conocimiento" es una herramienta indispensable para enfrentar los nuevos desafios que impone el mundo globalizado. Constituyéndose un canal continuo de difusión de la información entre los trabajadores, los clientes y la sociedad en general.

Las Organizaciones empresariales peruanas, en las últimas décadas se han visto en crisis económica, social y política; sin embargo, no escapan de la globalización y la competitividad. Por lo tanto, se requiere mayor preparación y capacitación de los integrantes de la Organización, dicho objetivo se podrá lograr en forma rápida y efectiva a través de la "Gestión del Conocimiento"; es decir, que los conocimientos de la empresa se puedan transmitir entre todos los miembros de la Organización, a través del INTERNET, hacer accesible dicha información a los clientes y proveedores a través del EXTRANET, y a la sociedad en general mediante el INTERNET.

El empresario nacional debe entender que para ser competitivo tanto en el mercado nacional como internacional, dentro de economias globalizadas, debe gestionar empresas con un adecuado manejo del "conocimiento". Es por ello, que al conceptuar la gestión del conocimiento planteamos que es kel conjunto de procesos y sistemas que permiten que el Capital Intelectual de una organización aumente de forma significativa, mediante la gestión de sus capacidades de resolución de problemas de forma eficiente (en el menor espacio de tiempo posible), con el objetivo final de generar ventajas competitivas sostenibles en el tiempo".

\section{LA INFORMACIÓN CONTABLE Y LA GESTIÓN DEL CONOCIMIENTO}

Los nuevos sistemas de información y las tecnologias modernas no sólo procesan información sino que gestionan "conocimiento". El enfoque del Knowledge Management es la base que integra otros sistemas para facilitar el desarrollo, almace-namiento y flujo de conocimiento a lo largo de toda la organización. Son sistemas que facilitan la comunicación, rompiendo barreras espaciales, temporales y organizativas, que cambian la propia concepción de la empresa y de su cultura.

La última concepción es la de "Gestión por Competencias", que procede del ámbito de los Recursos Humanos, y que busca la gestión de las personas a través de sus competencias, es decir, teniendo en cuenta sus capacidades, habilidades y conocimientos.

Un sistema de información de recursos humanos utiliza como fuentes de datos los elementos suministrados por:

- Base de datos de recursos humanos.

- Reclutamientos y selección de personal.

- Entrenamiento y desarrollo de personal.

- Evaluación del desempeño.

- Administración de salarios.

- Registros y control de personal, respecto de fallas, atrasos, disciplinas, etc.

- Estadísticas de personal.

- Higiene y seguridad.

- Jefaturas respectivas, etc. 
El sistema de información de Recursos Humanos debe planearse e implementarse para alcanzar determinados objetivos. El logro de estos objetivos es lo que permitirá evaluar la eficacia del sistema: hacer que los organismos de línea puedan tomar decisiones adecuadas respecto de las personas. Bajo esta concepción se sitúan las organizaciones que aprenden (Learning Organizations). Las empresas de hoy en día no pueden sobrevivir sin aprender continuamente, para la que hace falta una cultura de aprendizaje permanente.

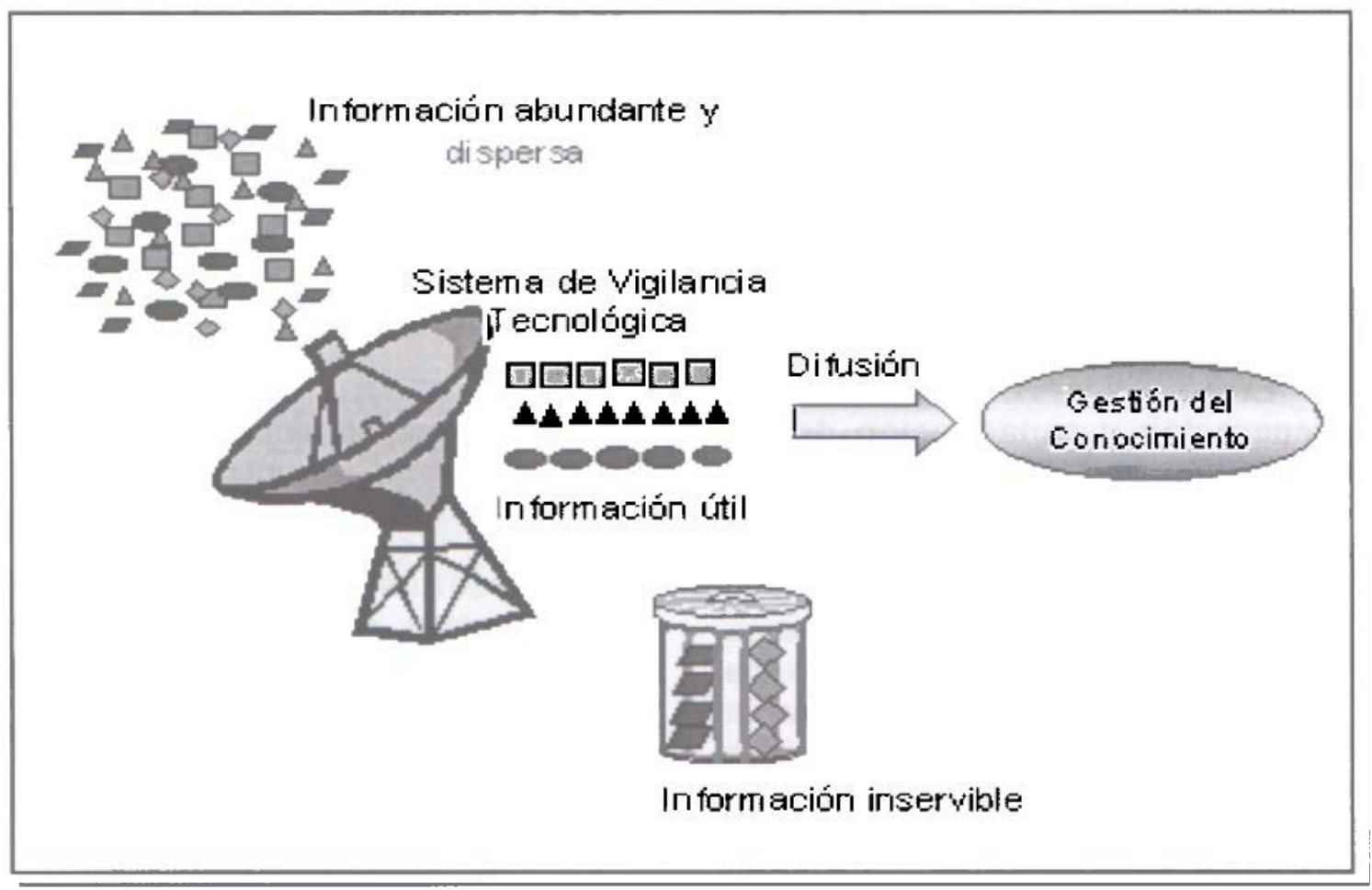

La Gestión del Conocimiento propicia el intercambio y generación del conocimiento que junto con la creatividad de las personas son el pilar para la innovación. También supone una mayor eficacia en el trabajo de las personas, eliminando tareas de poco valor añadido, aumentando su productividad.

Lo que brinda las ventajas competitivas de la Gestión del Conocimiento no es la cantidad de conocimientos que se consigan reunir y almacenar, sino más bien el uso que se haga de él. Para ello, es necesaria la adopción de una cultura corporativa que celebre el intercambio y la colaboración.

Una de las fuentes principales para la generación de conocimiento en la empresa es la Vigilancia Tecnológica. La Vigilancia puede tener identidad y vida propia dentro de la organización, pero es bajo el paraguas de la Gestión del Conocimiento cuando queda integrada totalmente dentro de los procesos de negocio de la empresa. La Vigilancia consiste en captar información del entorno, seleccionar la que se considere relevante para el negocio, difundirla en el seno de 
su Organización y utilizarla como herramienta en la toma de decisiones, es decir, un sistema organizado de observación y análisis del entorno, seguido de una eficaz circulación interna y utilización de la información para la toma de decisiones.

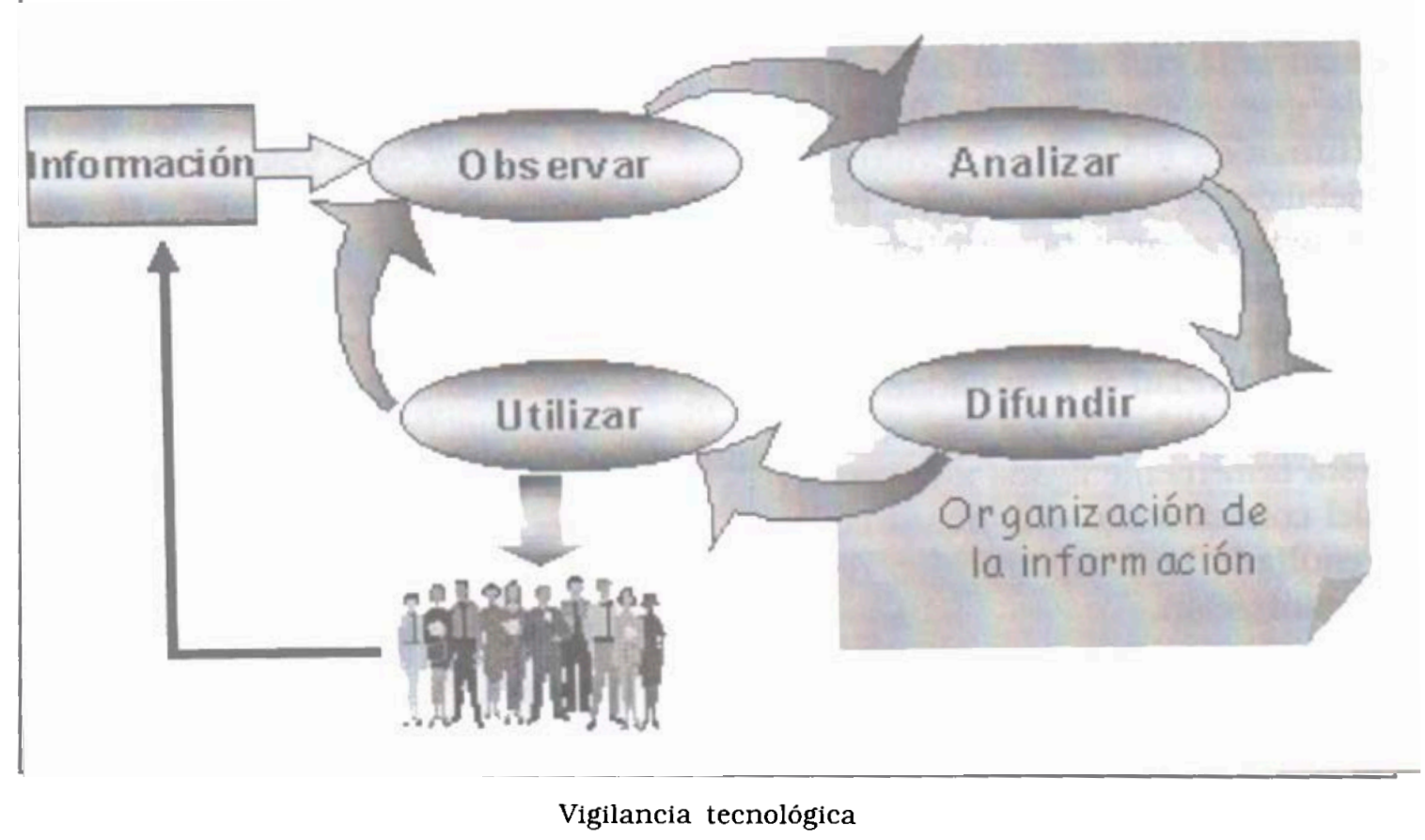

Al finalizar este articulo podemos manifestar que ésta es una extraordinaria oportunidad para los profesionales de la información contable y los profesionales de Gestión-Empresarial, para que puedan aportar sus conocimientos y su experiencia en la difusión y explotación de la información; permitiendo ofrecer a clientes, empleados y proveedores unas técnicas $y$ herramientas que aumentarân su capacidad de productividad y liderazgo en sus respectivas organizaciones.

\section{CONCLUSIONES}

1. El sistema de informaciôn de las empresas debe estar integrada a los procesos de negocios de la empresa, la misma debe captar los aspectos y/o hechos más relevantes del entorno próximo o remoto, la cual debe ser seleccionada para luego difundirla en el seno de su Organización y utilizarla como herramienta en la toma de decisiones, es decir, todo un sistema organizado de gestión del conocimiento.

2. La contabilidad debe proveer información cuantitativa $y$ cualitativa sobre los intangibles que conforman el capital intelectual de la empresa $y$ reconocer a éstos en los estados financieros utilizando para ello la normatividad dispuesta en la NIC 38 y la aplicación de uno de los modelos que presentamos, tales como: Skandia, Intellectual Assets Monitor, EVA y Valor Intangible Calculado, que poseen una perspectiva contable mucho más amplia ya que basan su 
estudio en la diferencia que existe entre el valor de mercado y el varlor de la compañia en libros.

3. La contabilidad debe informar los aspectos cualitativos y cuantitativos de los recursos humanos que maneja la entidad, con la finalidad de que la Gerencia de Recursos Humanos identifique, evalúe las debilidades y problemas en forma oportuna con la finalidad de plantear alternativas y soluciones eficaces que no mellen la productividad y estabilidad de la empresa, toda vez que esta variable está firmemente ligada a la gestión del conocimiento que busca como resultado la competitividad de la organización.

4. Al finalizar el presente artículo pudimos constatar que la gran mayoria de los empresarios en el Perú están conscientes que la complejidad del entorno actual, saturado de competencia, de problemas sociales, de clientes exigentes, e inmerso en un creciente proceso de globalización, hace necesario más que nunca el pensar y repensar en las organizaciones, darles sentido de dirección, rediseñar u optimizar los procesos existentes, desarrollar estructuras organizacionales aptas para que los procesos productivos funcionen utilizando agresivamente la tecnologia de punta que ayude a materializar la visión trazada para la organización.

\section{BIBLIOGRAFÍA}

1. G. ARTECHE, W. Rozas. "Conocimiento Estratégico; crear Valor con la Gestión del Conocimiento". Revista. Harvard Deusto Business Review, 1999.

2. CARL DULMAN y Colaboradores. Informe sobre el Desarrollo Mundial: El Conocimiento al servicio del Desarrollo. Ed. Mundi Prensa, Madrid, 1999.

3. Alvin Toffler. La Tercera Ola. Editores Plaza \& Janes S.A. Barcelona, 1980.

4. Peter Drucker. Lagerencia del Futuro. Grupo Editorial Norma. España, 1993.

5. George S. Yip. Glabalización Estrategias para obtener una ventaja competitiva internacional. Grupo Editorial Norma. España, 1993.

6. Arias Galicia, Fernando. Administración de Recursos Humanos. Ed. Trillas. México, 1994.

7. Fernández, E.; Montes, J.M.; Vázquez, C.J. (1998), Los Recursos Intangibles como Factores de Competitividad de la Empresa. Dirección y Organización. N. ${ }^{\circ} 22$

8. Jiménez, A. 1999. "Las Competencias y el Capital Intelectual: La manera de gestionar personas en la Era del Conocimiento", Boletín Club Intelec, abril, N. ${ }^{\circ}$ 2, pp. 2-5, Euroforum, Madrid, 